\title{
ON THE GEOMETRY OF STREAMLINES IN HYDRO- MAGNETIC FLUID FLOWS WHEN THE MAGNETIC FIELD IS ALONG A FIXED DIRECTION
}

\author{
E. R. SURYANARAYAN
}

1. Introduction. In this paper we discuss three-dimensional steady flows of inviscid magnetic fluids. We assume that the magnetic field is along a fixed direction. The main purpose is to investigate various dynamical and kinematical relations connecting the flow and the field quantities with the geometrical parameters of the streamlines. The expressions for the tangent, principal normal and binormal vectors and the curvature and torsion of the streamlines are given in terms of the velocity components, the pressure, the density and the magnitude of the magnetic field. The variations of the hydromagnetic pressure along the streamlines, along their principal normals and along their binormals are obtained. It is observed that the hydromagnetic pressure along the binormals remains constant. The Bernoulli function is defined and it is found that if this function exists then the Bernoulli surfaces contain both the streamlines and the vortex lines. We shall determine an intrinsic relation satisfied by the Bernoulli function. From this relation we obtain a necessary and sufficient condition for the Bernoulli surfaces to be a family of parallel surfaces. We find that the Bernoulli surfaces exist in the case of incompressible fluid and they form the surfaces on which the sum of the fluid pressure, the kinetic energy and the magnetic energy is constant. In the case of isentropic flows, the variation of the fluid pressure along the streamline is expressed in terms of the Mach number, the magnetic field, the divergence of the unit vector along the streamlines, the density and the magnitude of velocity. In the three-dimensional nonmagnetic gas flows this result reduces to the result of Kanwal [1].

Finally, we shall obtain a class of circular helical flows.

2. The equations. Let $x^{j}(j=1,2,3)$ denote the variables of a system of Cartesian orthogonal coordinates. In order to obtain the summation convention, we shall write indices in covariant and contravariant positions. We shall write $\partial_{i}=\partial / \partial x^{i}$. The equations of continuity and motion and the Maxwell relations for an inviscid, conductive fluid are [2]

Received by the editors August 8, 1963. 


$$
\begin{aligned}
\partial_{i}\left(\rho u^{i}\right) & =0 \\
\rho u^{j} \partial_{j} u_{i}+\partial_{i} P-\eta H^{j} \partial_{j} H_{i} & =0 \\
u^{j} \partial_{j} H_{i}-H^{j} \partial_{j} u_{i}+H_{i} \partial_{j} u^{j} & =0 \\
\partial_{i} H^{i} & =0
\end{aligned}
$$

where $\eta$ is the magnetic permeability; $P=p+(1 / 2) \eta H^{2}$ is the hydromagnetic pressure, $p$ being the fluid pressure, $H$ the magnitude of the magnetic field $H^{i} ; u^{i}$ is the velocity vector and $\rho$ is the density.

3. The basic decomposition. If $s^{i}$ denotes the unit tangent vector along the direction of $u^{i}$, then we may write

$$
u^{i}=q s^{i} .
$$

Since we are assuming that $H^{i}$ is along a constant direction we write

$$
H^{i}=B h^{i}
$$

where $h^{i}$ is a constant unit vector. Substituting for $u^{i}$ from the equation (3.1) into the equation (2.1), the equation (2.1) becomes

$$
\partial_{i} s^{i}+s^{i} \partial_{i}(\mathrm{l} \iota \rho q)=0 .
$$

Substituting for $u^{i}$ from the equation (3.1) and for $H^{i}$ from the equation (3.2) into the equation (2.2), the equation (2.2) reduces to

$$
\rho q s_{i} s^{j} \partial_{j} q+\rho q^{2} s^{j} \partial_{j} s_{i}+\delta_{i} P-\eta h_{i} h^{j} \partial_{j} H^{2}=0 .
$$

The expression $s^{j} \partial_{j} s_{i}$ appearing in the second term of the above equation is the curvature vector $k n_{i}, k$ being the curvature and $n_{i}$ the unit principal normal vector of the streamlines. Therefore, the equation (3.4) can be written in the form

$$
\rho q(d q / d s) s_{i}+\rho k q^{2} n_{i}+\partial_{i} P-\eta H(d H / d h) h_{i}=0,
$$

where we have written $s^{i} \partial_{i}=d / d s$ and $h^{i} \partial_{i}=d / d h$. By use of the equations (3.1) and (3.2), the equations (2.3) assume the form

$$
\begin{aligned}
q(d H / d s) h_{i}+B(d q / d h)+H q\left(d s_{i} / d h\right) & \\
& +B\left[(d q / d s)+q \partial_{j} s^{j}\right] h_{i}=0 .
\end{aligned}
$$

Again, the equations (2.4) and (3.2) imply that

$$
d H / d h=0 \text {. }
$$

Let us now take the scalar product of the equations (3.5) with $s^{i}, n^{i}$ and $b^{i}$, where $b^{i}$ is the unit binormal vector of the streamlines, and make use of the orthogonal conditions of these vectors. Then (3.5) becomes 


$$
\begin{aligned}
\rho q(d q / d s)+d P / d s & =0 \\
\rho q^{2} k+d P / d n & =0, \\
d P / d b & =0
\end{aligned}
$$

where $d / d n$ and $d / d b$ denote the directional derivative operators along the principal normal and the binormal, respectively. Again taking the scalar product of the equations (3.6) with $s^{i}, n^{i}, b^{i}$, respectively, we obtain

$$
\begin{aligned}
h_{i} s^{i}[d(H q) / d s+q H \mu]+H(d q / d h) & =0, \\
h_{i} n^{i}[d(H q) / d s+q H \mu]+H q n^{i}\left(d s_{i} / d h\right) & =0, \\
h_{i} b^{i}[d(H q) / d s+q H \mu]+H q b^{i}\left(d s_{i} / d h\right) & =0,
\end{aligned}
$$

where $\mu$ is the divergence of $s^{i}$. The equation (3.3) now becomes

$$
d(\ln q \rho) / d s=-\mu .
$$

The equations (3.7)-(3.14) are the intrinsic formulations of the equations (2.1)-(2.4). The equations (3.9) and (3.10) imply that the hydromagnetic pressure does not vary along the binormal and its variation along the principal normal depends on the curvature of the streamlines. The formulas (3.8), (3.9) and (3.10) are well known in the three-dimensional fluid flows in the nonmagnetic case [3]. From the equation (3.9) we have that in the case of straight line flows the hydromagnetic pressure does not vary along the principal normal and conversely.

Furthermore the unit binormal vector is given by

$$
b^{i}=e^{i j k} t_{j} n_{k},
$$

where $e^{i j k}$ denotes the permutation tensor with components $1,0,-1$ according to whether $(i j k)$ is an even permutation, contains a repeated integer, or is an odd permutation. Substituting for $s_{j}$ from the equation (3.1) and for $n_{j}$ from the equation (3.5) into the equation (3.15), the equation (3.15) becomes

$$
b^{i}=-\frac{1}{\rho k q^{3}} e^{i j k} u_{j} \partial_{k} P .
$$

Differentiating the above equation along the streamline and making use of the Frenét formula [4],

$$
d b^{i} / d s=-\tau n^{i},
$$

where $\tau$ is the torsion of the streamlines, we get the following relation connecting the torsion of the streamlines with the flow quantities and the magnitude of the field 


$$
\begin{aligned}
-\tau n^{i}=e^{i j k}\left[\frac{1}{k^{2} \rho^{2} q^{6}} \frac{d}{d s}(\rho k q) u_{j} \partial_{k} P\right. & \\
& \left.-\frac{1}{\rho k q^{8}}\left\{\partial_{k} P\left(d u_{j} / d s\right)-d\left(\partial_{k} P\right) / d s\right\}\right] .
\end{aligned}
$$

4. An intrinsic expression for the Bernoulli function. The equation of motion (2.2) can be transformed into the form [5]

(4.1) $-\rho e_{i j k} e^{k m n} u^{j} \partial_{m} u_{n}+\eta e_{i j k} e^{k m n} H^{j} \partial_{m} H_{n}=\partial_{i} P-\frac{1}{2} \rho \partial_{i} q^{2}-\frac{1}{2} \partial_{i}\left(\eta H^{2}\right)$.

And we have also

$$
e_{i j k} e^{k m n}=\delta_{i}^{m} \delta_{j}^{n}-\delta_{j}^{m} \delta_{i}^{n},
$$

where $\delta_{j}^{\ell}$ is the Kronecker delta. Substituting into the equation (4.1) for $u_{j}$ from the equation (3.1) and for $H_{j}$ from the equation (3.2), the equation (4.1) becomes, by use of (4.2),

(4.3) $-\rho q(d q / d b) b_{i}+\rho q[k q-(d q / d n)] n_{i}=-\partial_{i} P-\frac{1}{2} \rho \partial_{i} q^{2}$.

Let us now introduce the Bernoulli function $B$, if it exists, defined by

$$
\begin{aligned}
\partial_{i} B & =-\frac{1}{\rho}\left[\partial_{i} P+\frac{1}{2} \partial_{i}\left(\rho q^{2}\right)\right]+\frac{1}{2} q^{2} \partial_{i} \rho \\
& =-\frac{1}{\rho} \partial_{i}\left(p+\frac{1}{2} \eta H^{2}+\frac{1}{2} \rho q^{2}\right)+\frac{1}{2} q^{2} \partial_{i} \rho .
\end{aligned}
$$

If $d B / d \epsilon$ denotes the rate of change of $B$ with respect to a displacement normal to the Bernoulli surfaces $B=$ constant, we have, by use of the equations (4.3) and (4.4), that

$$
d B / d \epsilon=\left[q^{2}(d q / d b)^{2}+q^{2}\{k q-(d q / d n)\}^{2}\right]^{1 / 2} .
$$

Hence, a necessary and sufficient condition that the surfaces $B=$ constant consist of parallel surfaces is that

$$
q^{2}\left[(d q / d b)^{2}+(k q-d q / d n)^{2}\right]=\text { constant }
$$

along each surface of the family. This condition holds in the nonmagnetic case also [3]. Taking the scalar product of the equation (4.3) with $s^{i}$ we find that

$$
s^{i} \partial_{i} B=0 \text {. }
$$

And the vorticity vector is given by [3] (4.6) $w^{i}=\left[b^{k}\left(d t_{k} / d n\right)-n^{k}\left(d t_{k} / d b\right)\right] t^{i}+(d q / d b) n^{i}+[k q-(d q / d n)] b^{i}$. 
From the equations (4.3) and (4.6) we find that

$$
w^{i} \partial_{i} B=0 .
$$

The equations (4.5) and (4.7) imply that the Bernoulli surfaces contain both the streamlines and vortex lines. This result is well known for fluid flows in the nonmagnetic case [6].

If the fluid is incompressible the equation (4.4) yields that

$$
\partial_{i} B=-\frac{1}{\rho} \partial_{i}\left(p+\frac{1}{2} \eta H^{2}+\frac{1}{2} \rho q^{2}\right)=-\frac{1}{\rho} \partial_{i}(p+E),
$$

where $E$ is the sum of the magnetic and kinetic energies. Hence, if the fluid is incompressible the Bernoulli surfaces do exist and they constitute a family of surfaces $p+E=$ constant.

If the fluid is isentropic, we then have $d p / d \rho=a^{2}$, where $a$ is the local sound speed and the equation (4.4) can be written in the form

$$
\partial_{i} B=-\frac{1}{\rho}\left[\partial_{i} E+\frac{1}{2} q^{2}\left(1-2 M^{2}\right) \partial_{i} \rho\right],
$$

where $M$ is the Mach number and is equal to $q / a$. Taking the scalar product of the equation (4.8) with $s^{i}$ and making use of the result (4.5) we find that

$$
2(d E / d s)=-q^{2}\left(1-2 M^{2}\right)(d \rho / d s) .
$$

The above equation implies that the sum of the kinetic and magnetic energies does not vary along the streamlines if the density does not vary along the streamlines or if $M=(1 / \sqrt{ } 2)$ and conversely.

We shall now obtain a generalization of the result obtained by Kanwal for the three-dimensional gas flows in the nonmagnetic case [1]. Solving for $\partial_{i} \ln \rho$ in (2.2) and substituting into (2.1) we obtain

$$
\partial_{i} u^{i}=\left(u^{i} / a^{2}\right)\left[(\eta / \rho) H^{j} \partial_{j} H_{i}-(\eta / 2 \rho) \partial_{i} H^{2}-u^{j} \partial_{j} u_{i}\right]=0
$$

in the isentropic case. Substituting in the left-hand side for $u^{i}$ from (3.1) and for $H^{i}$ from (3.2) we find that (4.9) becomes

$$
\partial_{i} u^{i}=\left(H q \eta / \rho a^{2}\right)(d H / d s)+M^{2}(d q / d s) .
$$

From the equations (2.1) and the equation (3.14) we find that

$$
\partial_{i} u^{i}=-\mu q-(q / \rho)(d \rho / d s), \quad d q / d s=-(q / \rho)(d \rho / d s) .
$$

The equations (4.10) and (4.11) imply that

$$
\frac{1}{\rho q^{2}} \frac{d p}{d s}=\frac{1}{M^{2}-1}\left(\frac{H \eta}{q^{2} \rho} \frac{d H}{d s}+\mu\right)
$$


which in the nonmagnetic case reduces to the result obtained by Kanwal.

5. Helical flows. The streamlines of the flow are along the helices on coaxial cylinders. Such flows have the property $\mu=\partial_{i} s^{i}=0$ [7]. We will introduce the cylindrical coordinates $r, \theta, z$ and write

$$
s_{i}=\theta_{i} \sin \beta+z_{i} \cos \beta, \quad h_{i}=z_{i},
$$

where $\theta_{i}$ and $z_{i}$ are unit vectors along the increasing $\theta$ and the $z$ directions, respectively. The angle $\beta$ of the helices is, in general, a function of $r$. The helices form geodesics on the cylinders $r=$ constant, that is, the principal normal of a helix is along the normal to these cylinders. With this property, and by use of the Euler's equation for the normal curvature of a curve to evaluate $k$, the curvature of the helix [8], we have

$$
k=\sin ^{2} \beta / r \text {. }
$$

We have also that

$$
b_{i}=\theta_{i} \cos \beta-z_{i} \sin \beta, \quad n_{i}=r_{i}, \quad d s_{i} / d z=0,
$$

where $r_{i}$ is the unit vector along the radius of the cylinder. By use of the equations (5.1)-(5.3), the equations (3.7)-(3.14) now become

$$
\begin{aligned}
q \rho\left[\frac{\sin \beta}{r} \frac{\partial q}{\partial \theta}+\cos \beta \frac{\partial q}{\partial z}\right]+\frac{\sin \beta}{r} \frac{\partial P}{\partial \theta}+\cos \beta \frac{\partial P}{\partial z} & =0, \\
\rho q^{2}\left(\sin ^{2} \beta / r\right) & =\partial P / \partial r \\
\frac{\cos \beta}{r} \frac{\partial P}{\partial \theta}-\sin \beta \frac{\partial P}{\partial z} & =0 \\
\frac{\sin \beta}{r} \frac{\partial}{\partial \theta}(H q)+\cos \beta \frac{\partial}{\partial z}(H q) & =0 \\
\frac{\sin \beta}{r} \frac{\partial}{\partial \theta}(\rho q)+\cos \beta \frac{\partial}{\partial z}(\rho q) & =0 .
\end{aligned}
$$

The above equations constitute the equations governing the motion when the streamlines are helices.

Now let us find a class of solutions for which $H, q, P, \rho$, are all functions of $r$ only. In this case we have to satisfy the equation (5.6) which can now be written in the form 


$$
d P / d r=\rho q^{2}\left(\sin ^{2} \beta / r\right) .
$$

The above condition gives all the helical flows with the flow and field parameters as functions of $r$. As a special case, consider a flow with

$$
P=1-e^{-r}, \quad q^{2}=2 r e^{-r} .
$$

These equations imply that $\beta=\pi / 4$.

\section{REFERENCES}

1. R. P. Kanwal, Variction of flow quantities along streamlines and their principal normals and binormals in three-dimensional gas flows, J. Math. Mech. 6 (1957), 621628.

2. S. Chandrasekhar, Hydrodynamic and hydromagnetic stabitity, p. 148, Clarendon Press, Oxford, 1961.

3. $\mathrm{N}$. Coburn, Intrinsic relations satisfied by the vorticity and velocity vectors in fluid flow theory, Michigan Math. J. 1 (1952), 113-130.

4. C. E. Weatherburn, Differential geometry of three dimensions. Vol. I, p. 15, Cambridge Univ. Press, Cambridge, 1955.

5. S. Chandrasekhar, Hydrodynamic and hydromagnetic stability, p. 157, Clarendon Press, Oxford, 1961.

6. H. Lamb, Hydrodynamics, p. 244, Dover, New York, 1945.

7. C. E. Weatherburn, Differential geometry of three dimensions, Vol. I, p. 258, Cambridge Univ. Press, Cambridge, 1955.

8. - Differential geometry of three dimensions, Vol. I, p. 73, Cambridge Univ. Press, Cambridge, 1955.

UNIVERSITY OF RHODE ISLAND 